\title{
Impaired Fibrinolysis in Angiographically Documented Coronary Artery Disease
}

\author{
Adriano Basques Fernandes, ${ }^{1}$ Luciana Moreira Lima, ${ }^{1}$ \\ Marinez Oliveira Sousa, ${ }^{1}$ Vicente de Paulo Coelho Toledo, ${ }^{1}$ Rashid Saeed Kazmi, \\ Bashir Abdulgader Lwaleed, ${ }^{3}$ and Maria das Graças Carvalho' \\ ${ }^{1}$ Faculty of Pharmacy, Federal University of Minas Gerais, Avenida Antonio Carlos 6627, 31270-901 Belo Horizonte, MG, Brazil \\ ${ }^{2}$ Department of Haematology, University Hospital Southampton, Southampton, UK \\ ${ }^{3}$ Faculty of Health Sciences, University of Southampton, Southampton, UK
}

Correspondence should be addressed to Rashid Saeed Kazmi; rashid.kazmi@uhs.nhs.uk

Received 13 October 2014; Revised 27 January 2015; Accepted 27 January 2015

Academic Editor: Owen McCarty

Copyright (C) 2015 Adriano Basques Fernandes et al. This is an open access article distributed under the Creative Commons Attribution License, which permits unrestricted use, distribution, and reproduction in any medium, provided the original work is properly cited.

\begin{abstract}
Impaired fibrinolysis may predispose to coronary artery disease (CAD). Hypofibrinolysis due to high levels of plasminogen activator inhibitor-1 (PAI-1) has been reported in CAD. A novel regulator of fibrinolytic activity, thrombin activatable fibrinolysis inhibitor (TAFI), has attracted attention in recent years. It acts by blocking the formation of a ternary complex of plasminogen, fibrin, and tissue plasminogen activator (t-PA). Previously ambiguous results regarding TAFI levels have been reported in CAD. We measured plasma levels of PAI-1 and TAFI antigen in 123 patients with age ranging from 40 to 65 years who had been submitted to coronary angiography and assessed the association of these markers with the extent of stenosis in three groups: angiographically normal artery (NAn), mild to moderate atheromatosis (MA), and severe atheromatosis (SA). Plasma levels of PAI-1 were increased in patients with severe atheromatosis compared to mild/moderate atheromatosis or to normal patients $(66.60,40.50$, and $34.90 \mathrm{ng} / \mathrm{mL}$, resp.; $P<0.001)$. For TAFI no difference was found between different groups. When patients were grouped in only two groups based on clinical cut-off point for intervention (stenosis less than or above 70\%) we found increased plasma levels for PAI-1 (37.55 and $66.60 \mathrm{ng} / \mathrm{mL}$, resp.; $P<0.001)$ and decreased plasma levels for TAFI $(5.20$ and $4.53 \mu \mathrm{g} / \mathrm{mL}$, resp.; $P=0.04)$ in patients with stenosis above $70 \%$. No difference was found in PAI-1 or TAFI levels comparing the number of affected vessels. Conclusion. As evidenced by a raised level of PAI-1 antigen, one can suggest an impaired fibrinolysis in stable CAD, although no correlation with the number of affected vessels was found. Curiously, a decreased plasma level of total TAFI levels was observed in patients with stenosis above $70 \%$. Further studies measuring functional TAFI are required in order to elucidate its association with the extent of degree of atheromatosis.
\end{abstract}

\section{Introduction}

The endothelium mediates a variety of vital physiological functions. While in health it maintains vascular integrity by expressing vasoprotective and thromboresistant molecules, on activation endothelial cells (ECs) acquire a phenotype that promotes atherosclerosis [1]. The thrombin catalyzed conversion of plasma fibrinogen into fibrin is the final step of coagulation cascade during haemostasis. The formation of thrombus is followed by the process of fibrinolysis which consists of an enzymatic dissolution of the fibrin clot by plasmin.
It is controlled by endothelial cells through secretion of physiological plasminogen activators like tissue type plasminogen activator (t-PA) and urokinase type plasminogen activator (u-PA). Fibrinolysis is initiated when both plasminogen and t-PA bind to fibrin surface to generate plasmin [2]. However, plasmin is generated on the surface of endothelial cells in the presence or absence of fibrin. In the absence of fibrin plasmin generation is dependent on the constitutively expressed plasminogen and t-PA receptor, annexin $2 \mathrm{~A}$. The endothelium also exerts an inhibitory effect on fibrinolysis through the synthesis of an inhibitor, type 1 plasminogen 
activator inhibitor (PAI-1). High levels of PAI-1 have been shown to be associated with CAD [3, 4]. Yet another fibrinolytic inhibitor is thrombin activatable fibrinolysis inhibitor (TAFI), a plasma zymogen that potently inhibits fibrinolysis when converted to an active enzyme by thrombin, plasmin, trypsin, and, more efficiently, thrombin-thrombomodulin complex [5]. Activated TAFI (TAFIa) inhibits fibrinolysis by removing the carboxyterminal lysine (and arginine) residues on partially degraded fibrin, blocking the formation of a ternary complex of plasminogen, and t-PA. Some studies have shown a trend for increased TAFI levels in CAD patients [6$8]$. On the contrary other investigators have found decreased levels of TAFI in CAD patients [9].

The aim of this study was to investigate the association of PAI-1 and TAFI antigen levels with increasing degrees of coronary atheromatosis in patients undergoing angiography.

\section{Material and Methods}

The population investigated consisted of 123 subjects with age ranging from 40 to 65 years, who had been consecutively submitted to coronary angiography in the Department of Haemodynamics of Socor Hospital, Belo Horizonte, Brazil. This protocol was submitted to the local ethical committees in research of Socor Hospital and Federal University of Minas Gerais. Signed informed consent was required for all subjects enrolled in this study.

This study assessed a population of intermediate to high risk of $\mathrm{CAD}$ who had been referred for catheterization due to worsening clinical features on a background of a history of stable angina. Patients with acute coronary syndrome in the preceding 3 months were excluded from the study as were those having concomitant treatment with anticoagulants, lipid lowering drugs, or estrogens, patients with known bleeding or thrombotic disorders, and those with renal, hepatic, autoimmune, or malignant diseases.

Coronary angiography was performed in all 123 subjects by percutaneous transfemoral approach. The images were recorded digitally and all angiograms were analyzed by three experienced cardiologists. The patients were grouped according to the angiographic findings as follows: no stenosis (Group I), stenosis of up to $30 \%$ of the luminal diameter in at least one coronary artery (Group II), stenosis of 30 to $70 \%$ of the luminal diameter in at least one coronary artery (Group III), and stenosis of more than $70 \%$ of the luminal diameter in at least one coronary artery (Group IV).

Blood samples were collected from $12 \mathrm{~h}$ fasting patients after coronary angiography (since patients stratification was dependent on this procedure), however, before any other intervention following angiography, into vacuum tubes containing $3.2 \mathrm{w} / \mathrm{v}$ sodium citrate as anticoagulant. Blood samples were immediately centrifuged at $2100 \mathrm{~g}$ for 20 minutes and plasma samples were separated and stored at $-70^{\circ} \mathrm{C}$ until analysis.

Plasma level of TAFI was performed using a commercially available ELISA Kit (TAFI Antigen Kit, Affinity Biologicals Inc., Canada) according to the manufacturer's instructions.
Quantitative determination of plasma TAFI was performed using citrated set diagnostic Visualize TAFI Antigen Kit (Affinity Biologicals Inc., Canada) whose analytical principle is the enzyme-linked immunosorbent assay (ELISA capture), strictly following the instructions provided by the manufacturer. A microplate reader, BIO-RAD 550-USA, was used for reading the reaction. The reference curve was performed using the standard provided by the manufacturer obtaining the points of $6.8,3.4,1.7,0.850,0.425$, and $0.213 \mathrm{mg} / \mathrm{mL}$ and two plasma controls provided by the kit were used to verify the assay performance. The concentration of TAFI in the sample was obtained using the following equation:

$$
\log (y)=A+B x \log (x)
$$

with reference value from 5.8 to $10.0 \mu \mathrm{g} / \mathrm{mL}$.

PAI-1 level was determined using a commercial ELISA Kit (IMUBIND PAI-1 ELISA Kit, American Diagnostica Inc., USA). The intra- and interassay coefficients were $9.0 \%$ and 6.6\%, respectively (IMUBIND PAI-1 ELISA Kit, American Diagnostica Inc., USA). For both, TAFI and PAI-1, control plasmas were used to verify the assay performance.

Normal distribution of the data was checked by ShapiroWilks test. The results were presented as mean and standard deviation (SD) when normally distributed and otherwise as median and interquartile ranges (25th and 75th percentiles). For normally distributed data, ANOVA was used to compare three groups. Mann-Whitney and Kruskal-Wallis tests followed by Dunn's test were used in case of non-normally data for comparison of three groups. Statistical analysis was performed by Sigma Stat version 1.0 software system. A value of $P<0.05$ was chosen for statistical significance.

\section{Results}

The baseline characteristics of the study participants are presented in Table 1. The three groups showed homogeneity in relation to age, sex, and body mass index and no statistically significant difference was noted. The incidence for hypertension was high in all three groups. The differences in the incidence of smoking, sedentary lifestyle, and previous history of CAD were statistically significant amongst different degrees of severity of CAD.

Plasma levels of PAI-1 were increased in patients with severe atheromatosis (Group IV) compared to mild/moderate atheromatosis (Groups II/III) $(P<0.001)$ or to angiographically normal (Group I) patients. For TAFI no statistically significant difference was found between groups. Plasma levels of PAI-1 and TAFI are presented in Table 2.

Considering that a clinical intervention must be made in all patients presenting with stenosis above $70 \%$, all of them were rescored in two groups: stenosis of up to $70 \%$ of the luminal diameter in at least one coronary artery and stenosis of more than $70 \%$ of the luminal diameter in at least one coronary artery. Table 3 shows results for PAI-1 and TAFI in these two groups.

Based on this clinical cut point for intervention, we found increased plasma levels for PAI-1 $(P<0.001)$ and decreased 
TABLE 1: Baseline patients' characteristics.

\begin{tabular}{|c|c|c|c|c|}
\hline & Group I & Groups II/III & Group IV & $P$ \\
\hline$n(\mathrm{M} / \mathrm{F})$ & $35(16 / 19)$ & $31(17 / 14)$ & $57(31 / 26)$ & ns \\
\hline Men & $16(45.7 \%)$ & $17(54.8 \%)$ & $31(54.4 \%)$ & ns \\
\hline Age (years) & $59.0 \pm 7.5$ & $59.5 \pm 9.0$ & $60.5 \pm 8.8$ & ns \\
\hline $\mathrm{BMI}\left(\mathrm{Kg} / \mathrm{m}^{2}\right)$ & $25.3 \pm 4.1$ & $26.8 \pm 4.7$ & $25.8 \pm 3.5$ & ns \\
\hline Current smoker & $6(17.1 \%)$ & $8(25.8 \%)$ & $23(40.4 \%)^{\mathrm{a}}$ & $P^{\mathrm{a}}=0.020$ \\
\hline Hypertension & $31(88.6 \%)$ & $25(80.6 \%)$ & $48(84.2 \%)$ & ns \\
\hline Sedentary lifestyle & $33(94.3 \%)$ & $23(74.2 \%)^{\mathrm{A}}$ & $43(75.4 \%)^{\mathrm{a}}$ & $\begin{array}{l}P^{\mathrm{a}}=0.021 \\
P^{\mathrm{A}}=0.023\end{array}$ \\
\hline Family history of CAD & $14(40.0 \%)$ & $18(58.1 \%)$ & $29(50.8 \%)$ & ns \\
\hline Diabetes mellitus & $5(14.3 \%)$ & $7(22.6 \%)$ & $8(14.0 \%)$ & ns \\
\hline Previous history of CAD & $7(20.0 \%)$ & $12(38.7 \%)$ & $35(61.4 \%)^{\mathrm{a}}$ & $P^{\mathrm{a}}<0.0001$ \\
\hline
\end{tabular}

$n$ = sample size; $\mathrm{M}$ = male; $\mathrm{F}$ = female; $\mathrm{BMI}=$ body mass index; ns = not significant; Group I = normal; Groups II/III = mild/moderate atheromatosis, up to $70 \%$ in at least one coronary artery; Group IV = severe atheromatosis, $>70 \%$ in at least one coronary artery; a/A = significant difference to Group I (ANOVA).

TABLE 2: Plasma fibrinolytic markers.

\begin{tabular}{|c|c|c|c|c|}
\hline & Group I & Groups II/III & Group IV & $P$ \\
\hline$n$ & 35 & 31 & 57 & - \\
\hline PAI-1 & $34.90(27.86 ; 42.43)$ & $40.50(34.24 ; 51.83)$ & $66.60^{\mathrm{a}, \mathrm{b}}(44.70 ; 91.65)$ & $P<0.001$ \\
\hline TAFI & $5.71 \pm 1.73$ & $5.21 \pm 1.42$ & $4.97 \pm 1.41$ & ns \\
\hline
\end{tabular}

$n=$ sample size; Group I = normal; Groups II/III = mild/moderate atheromatosis, up to $70 \%$ in at least one coronary artery; Group IV = severe atheromatosis, $>70 \%$ in at least one coronary artery; PAI- $1=$ type 1 plasminogen activator inhibitor; TAFI = thrombin activatable fibrinolysis inhibitor; ${ }^{\mathrm{a}}$ versus Group I and

b versus Groups II/III; ns = not significant.

Values for PAI-1 are given in median (25th and 75th percentiles) and expressed in $(\mathrm{ng} / \mathrm{mL})$.

Values for TAFI are given in mean \pm SD and expressed in $(\mu \mathrm{g} / \mathrm{mL})$.

TABLE 3: Plasma fibrinolysis markers considering stenosis of $70 \%$ as clinical cut point for intervention.

\begin{tabular}{lccc}
\hline & Stenosis $<70 \%$ & Stenosis $>70 \%$ & $P$ \\
\hline$n$ & 66 & 57 & - \\
PAI-1 & $37.55(30.90 ; 49.20)$ & $66.60^{\mathrm{a}}(44.70 ; 91.65)$ & $P<0.001$ \\
TAFI & $5.20(4.37 ; 6.31)$ & $4.53^{\mathrm{a}}(4.04 ; 5.63)$ & $P=0.04$
\end{tabular}

$n=$ sample size; PAI- $1=$ type 1 plasminogen activator inhibitor; TAFI $=$ thrombin activatable fibrinolytic inhibitor; ${ }^{a}$ versus stenosis $<70 \%$. Values for PAI-1 and TAFI are given in median (25th and 75th percentiles) and expressed in $\mathrm{ng} / \mathrm{mL}$ and $\mu \mathrm{g} / \mathrm{mL}$, respectively.

plasma levels for TAFI $(P=0.04)$ in patients with stenosis of more than $70 \%$.

We also explored the severity of CAD through the number of affected vessels in patients with stenosis of more than $70 \%$ versus plasma levels of PAI- 1 and TAFI. No statistically significant difference was found between groups (Table 4).

\section{Discussion}

Regulation of blood coagulation is an intricate coordination between different pathways. Maintaining a balance between thrombin-stimulated fibrin clot formation and plasmininduced clot lysis is essential for optimal haemostasis. Any disturbance in these pathways causes a haemorrhagic or a
TABle 4: Plasma fibrinolysis markers considering number of affected vessels.

\begin{tabular}{lcccc}
\hline & $1 \mathrm{v}$ & $2 \mathrm{v}$ & $3 \mathrm{v}$ & $P$ \\
\hline$n$ & 16 & 13 & 28 & - \\
PAI-1 & $92.79 \pm 60.45$ & $71.83 \pm 39.29$ & $62.15 \pm 31.97$ & $\mathrm{~ns}$ \\
TAFI & $5.31 \pm 1.52$ & $4.75 \pm 1.16$ & $4.87 \pm 1.46$ & $\mathrm{~ns}$ \\
\hline
\end{tabular}

$n=$ sample size; $1 \mathrm{v}=$ stenosis in one vessel; $2 \mathrm{v}=$ stenosis in two vessels; $3 \mathrm{v}$ $=$ stenosis in three or more vessels; PAI-1 = type 1 plasminogen activator inhibitor; TAFI $=$ thrombin activatable fibrinolytic inhibitor; $n s=$ not significant. Values for PAI- 1 and TAFI are given in mean \pm SD and expressed in $\mathrm{ng} / \mathrm{mL}$ and $\mu \mathrm{g} / \mathrm{mL}$, respectively.

thrombotic tendency depending on the shift of the balance [10]. The data from this study demonstrate that fibrinolysis is impaired in subjects with angiographically documented CAD. PAI-1 levels were increased in patients with severe atheromatosis compared to mild/moderate atheromatosis $(P<0.001)$ or angiographically normal patients.

Increased levels of PAI-1 have been described in patients with CAD after myocardial infarction (MI) [3] and are considered a risk factor for recurrence [4]. We tried to correlate the severity of disease with the number of affected vessels and PAI-1 levels, but no significant difference between different groups was found (Table 4). Thrombin also plays an important role in fibrinolytic modulation through activation 
of TAFI [11]. Activated TAFI inhibits plasmin formation and downregulates fibrinolysis [5] contributing to a hypofibrinolytic state in cardiovascular disease [9]. In the present study, we did not find any significant difference in TAFI levels between groups (Table 2). Interestingly, when we rescored groups based on clinical cut point for intervention (stenosis of more than 70\%) TAFI plasma levels were decreased in subjects with stenosis of more than $70 \%$ (Table 3) compared to those with stenosis of up to $70 \%$, consistent with a previous study [9]. No difference was found between severity of disease through number of affected vessels and TAFI levels (Table 4). Controversial results regarding TAFI levels in CAD patients have been described possibly because of both different characteristics of patients investigated and methods used for its determination [12].

Different clinical studies have investigated the possible relationship between TAFI and cardiovascular events [12]. The results have been inconsistent and studies have reported high, normal, and low plasma levels of TAFI [6-8].

We could speculate that the amount of TAFI generated during coagulation and fibrinolysis varies in different cardiovascular disease stages and, in addition, different results obtained through different assays demonstrate the variable reactivity of antibodies toward different isoforms of TAFI. An important study [13] comparing different TAFI assays has demonstrated that pro-TAFI assay measures high levels of TAFI in CAD, while TAFI antigen assays can detect no alterations in TAFI plasma levels or a slight decrease of TAFI plasma levels in the same samples. We can also speculate that different antibodies react to different isoforms of TAFI, yielding ambiguous results.

The importance of haemostatic alterations in CAD is being increasingly recognised in cardiovascular diseases. Our study has demonstrated that PAI-1 plasma levels are increased in a limited number of CAD patients in Brazilian patients, in line with previous studies $[5,9]$. Although decreased TAFI levels are in agreement with other studies [9], its physiological relevance and pathogenic role remain unclear since only the levels of TAFI antigen were evaluated precluding any conclusion on the TAFI function.

The major limitation of the present study was that our observations were based on a small number of patients, which precludes a specific cut-off for PAI-1 and TAFI antigens in severe disease. Another limitation is the fact that in a cohort cross-sectional study we tried to evaluate associations, not predictions or causation. Finally, our findings are related to a group of patients referred for catheterization due to thoracic pain selected by experienced cardiologists. Thus, our subjects with no stenosis may not be representative of the general population. Further work is required to investigate PAI- 1 and TAFI antigens roles in CAD in a greater number of patients and functional assays are also required for clarifying the real role of these fibrinolysis inhibitors. Also known modifiers of PAI-1 levels such as smoking, body mass index, and circadian variation should be considered for result interpretation.

In conclusion, this small study confirms an impaired fibrinolysis in stable CAD considering the increased levels of
PAI-I, indicating that this marker is actually associated with the atheromatosis extent, although the number of affected vessels seems not to have contributed to increase of PAI-1 levels.

\section{Conflict of Interests}

The authors declare that there is no conflict of interests regarding the publication of this paper.

\section{References}

[1] K. K. Wu, N. Aleksic, C. M. Ballantyne, C. Ahn, H. Juneja, and E. Boerwinkle, "Interaction between soluble thrombomodulin and intercellular adhesion molecule-1 in predicting risk of coronary heart disease," Circulation, vol. 107, no. 13, pp. 17291732, 2003.

[2] B. N. Bouma and L. O. Mosnier, "Thrombin Activatable Fibrinolysis Inhibitor (TAFI) at the interface between coagulation and fibrinolysis," Pathophysiology of Haemostasis and Thrombosis, vol. 33, no. 5-6, pp. 375-381, 2004.

[3] W. Koenig, D. Rothenbacher, A. Hoffmeister, M. Griesshammer, and H. Brenner, "Plasma fibrin D-dimer levels and risk of stable coronary artery disease: results of a large case-control study," Arteriosclerosis, Thrombosis, and Vascular Biology, vol. 21, no. 10, pp. 1701-1705, 2001.

[4] A. M. Thögersen, J.-H. Jansson, K. Boman et al., "High plasminogen activator inhibitor and tissue plasminogen activator levels in plasma precede a first acute myocardial infarction in both men and women: evidence for the fibrinolytic system as an independent primary risk factor," Circulation, vol. 98, no. 21, pp. 2241-2247, 1998.

[5] A. Paola Cellai, E. Antonucci, A. Alessandrello Liotta et al., "TAFI activity and antigen plasma levels are not increased in acute coronary artery disease patients admitted to a coronary care unit," Thrombosis Research, vol. 118, no. 4, pp. 495-500, 2006.

[6] A. Silveira, K. Schatteman, F. Goossens et al., "Plasma procarboxypeptidase $\mathrm{U}$ in men with symptomatic coronary artery disease," Thrombosis and Haemostasis, vol. 84, no. 3, pp. 364$368,2000$.

[7] V. Schroeder, T. Chatterjee, H. Mehta et al., “Thrombin activatable fibrinolysis inhibitor (TAFI) levels in patients with coronary artery disease investigated by angiography," Thrombosis and Haemostasis, vol. 88, no. 6, pp. 1020-1025, 2002.

[8] A. Santamaría, A. Martínez-Rubio, M. Borrell, J. Mateo, R. Ortín, and J. Fontcuberta, "Risk of acute coronary artery disease associated with functional thrombin activatable fibrinolysis inhibitor plasma level," Haematologica, vol. 89, no. 7, pp. 880881, 2004.

[9] I. Juhan-Vague, P. E. Morange, H. Aubert et al., "Plasma thrombin-activatable fibrinolysis inhibitor antigen concentration and genotype in relation to myocardial infarction in the North and South of Europe," Arteriosclerosis, Thrombosis, and Vascular Biology, vol. 22, no. 5, pp. 867-873, 2002.

[10] G. Cesarman-Maus and K. A. Hajjar, "Molecular mechanisms of fibrinolysis," British Journal of Haematology, vol. 129, no. 3, pp. 307-321, 2005.

[11] N. H. van Tilburg, F. R. Rosendaal, and R. M. Bertina, “Thrombin activatable fibrinolysis inhibitor and the risk for deep vein thrombosis," Blood, vol. 95, no. 9, pp. 2855-2859, 2000. 
[12] E. Ceresa, E. Brouwers, M. Peeters, C. Jern, P. J. Declerck, and A. Gils, "Development of ELISAs measuring the extent of TAFI activation," Arteriosclerosis, Thrombosis, and Vascular Biology, vol. 26, no. 2, pp. 423-428, 2006.

[13] M. Skeppholm, N. H. Wallén, K. Malmqvist, A. Kallner, and J. P. Antovic, "Comparison of two immunochemical assays for measuring thrombin-activatable fibrinolysis inhibitor concentration with a functional assay in patients with acute coronary syndrome," Thrombosis Research, vol. 121, no. 2, pp. 175-181, 2007. 


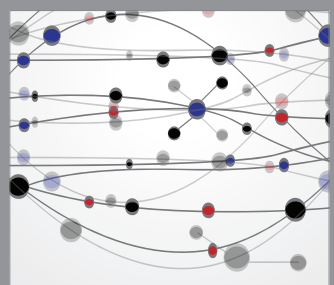

The Scientific World Journal
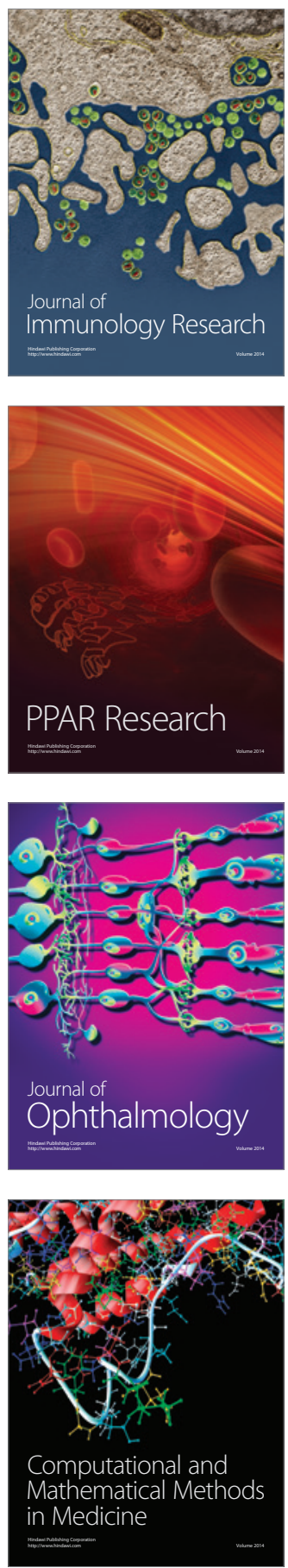

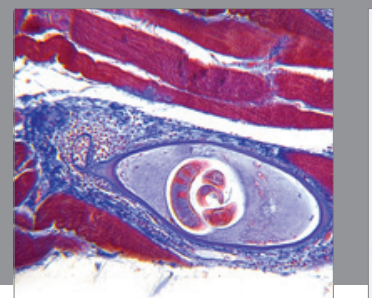

Gastroenterology

Research and Practice
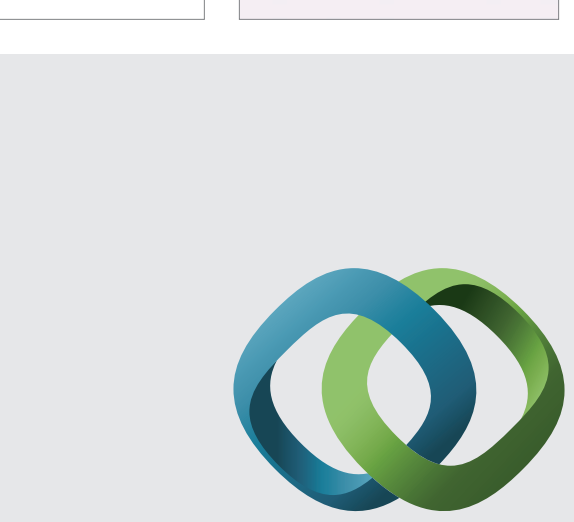

\section{Hindawi}

Submit your manuscripts at

http://www.hindawi.com
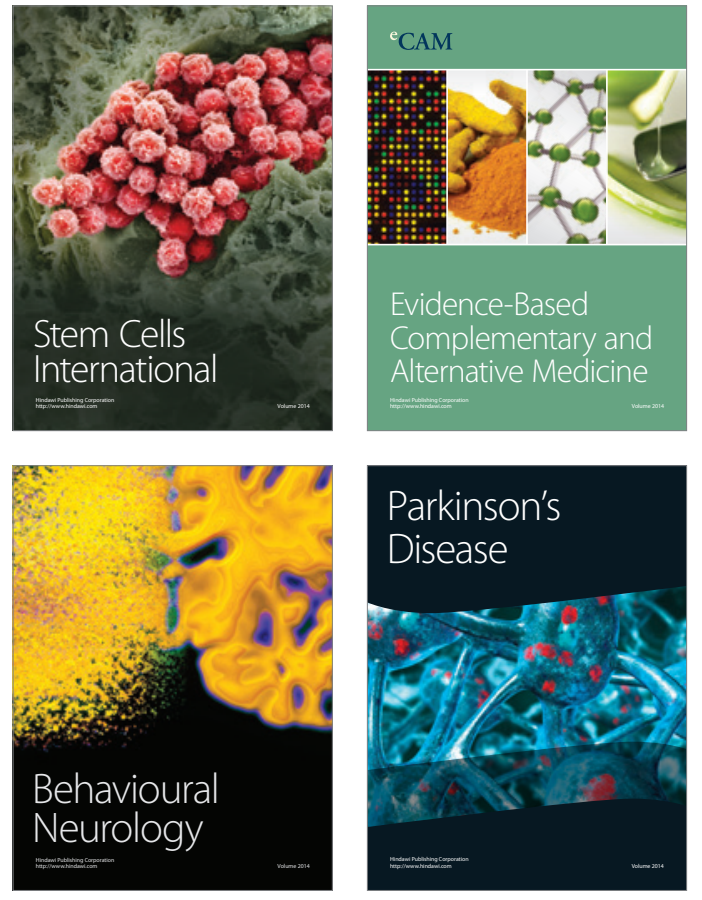
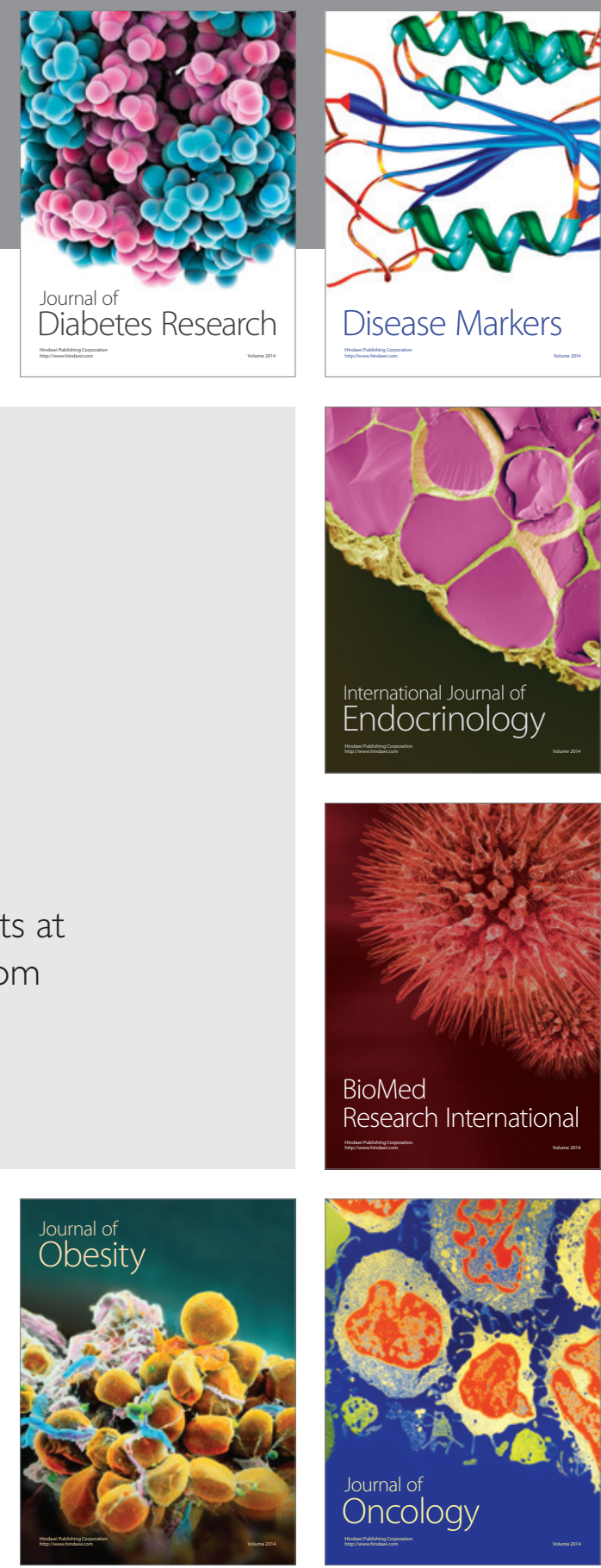

Disease Markers
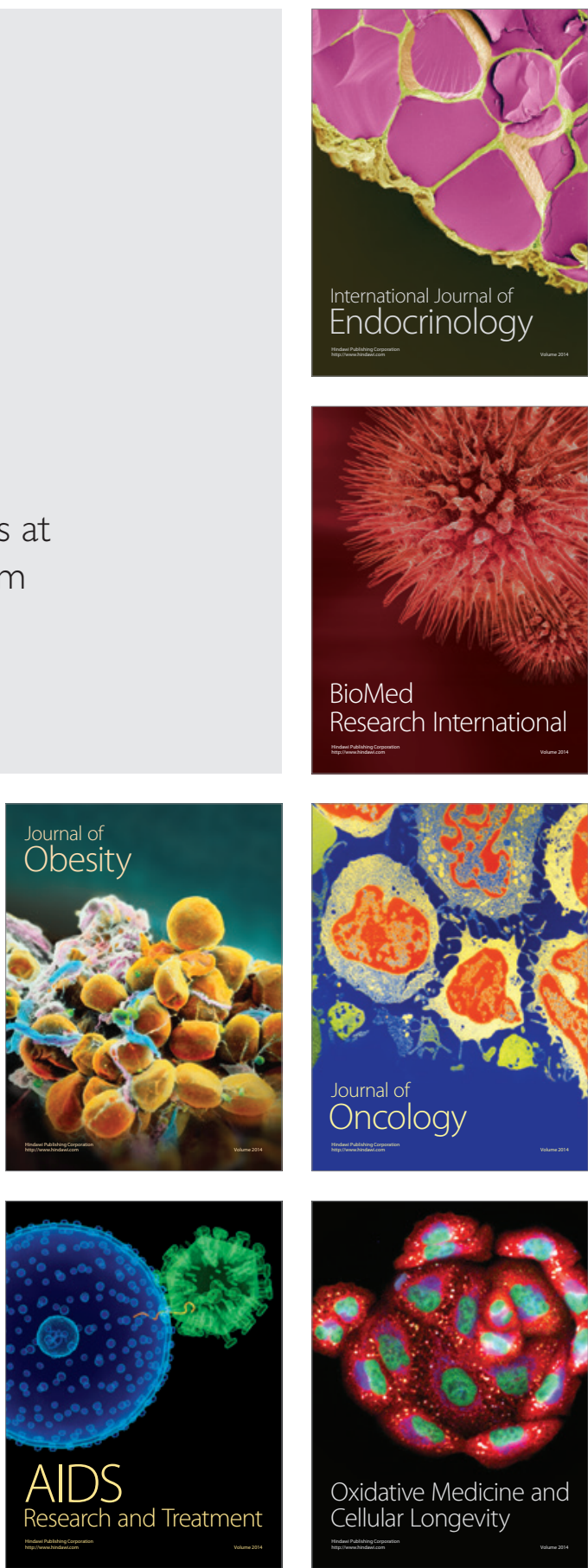